\title{
Surgical Reintervention in the Chin in a Patient with a Silicone Chin Implant - A Case Report
}

\author{
João Luiz Carlini ${ }^{1 *}$, Kendy Lipski², Silmara Regina Teodorovitz Roeder ${ }^{3}$ \\ ${ }^{1}$ Professor Cirurgia I-Federal University of Paraná, Curitiba, Brazil \\ ${ }^{2}$ Student Graduation in Federal University of Paraná, Curitiba, Brazil \\ ${ }^{3}$ Private Clinic, Curitiba, Brazil \\ *Corresponding Author: João Luiz Carlini, Professor Cirurgia I-Federal University of Paraná, Curitiba, Brazil; \\ Email: joaoluizcarlini@gmail.com \\ Received Date: 23-02-2021; Accepted Date: 15-03-2021; Published Date: 22-03-2021 \\ Copyright $^{\odot} 2021$ by Carlini JL, et al. All rights reserved. This is an open access article distributed under the terms \\ of the Creative Commons Attribution License, which permits unrestricted use, distribution and reproduction in \\ any medium, provided the original author and source are credited.
}

\begin{abstract}
The use of biomaterials may be an option for the treatment mainly of chin deficiencies. One of the most used materials is silicone prostheses. However, some problems with this material were observed, mainly the bone resorption by compression of the musculature, generating intense resorption, as detected in this described clinical case, where fracture of the jaw could occur. Prosthesis removal, chin osteotomy and reconstruction with an allogeneic graft were performed.
\end{abstract}

\section{Keywords}

Silicone Prostheses; Mentoplasty; Allogeneic Graft

\section{Introduction}

Chin surgery is an adjuvant procedure to define facial harmony. Its indication is for forward or backward movement of the chin, vertical augmentation or reduction of the chin, and for the correction of facial asymmetry. This procedure is important because it will provide bone support for the lower lip, reducing lower lip ptosis, improving the sealing of the lips and collaborating with facial harmony. 
Regarding the options for performing chin surgery, we can mention bone shaving, osteotomy and repositioning with fixation with miniplates and/or screws. In cases of chin deficiency, there is the option of using implants of materials such as polyethylene (MEDPOR) with a pre-molded shape and solid silicone. All of these options have advantages and disadvantages. Conventional osteotomies have the disadvantage of a new surgical site with osteotomy, with risk for the roots of the anterior teeth, paresthesia of the lower lip, and the need for fixation with steel wire, miniplates and/or screws. However, it offers greater stability of the result in the long term. Biomaterials like polyethylene prevent osteotomy, that is, there is less surgical trauma. However, their use requires skill. There is also a need to adapt the implant and fix it with screws. The material is expensive and there is a risk of contamination, in which case the implant would have to be removed.

An option, especially in patients with mandible deficiency (class II) and/or chin deficiency, is the use of solid silicone implants. The advantages of solid silicone implants are that they come in several sizes and shapes, they do not need fixation, and the procedure can be performed on an outpatient basis by intraoral or extraoral access. Some of the disadvantages are the cost of the material, the deformation and possible displacement of the implant, and especially the resorption of the symphyseal bone by the pressure that the soft tissue exerts on the material and that the material exerts on the bone.

The aim of this study was to demonstrate, through a clinical case, the harmful effect on the bone of a silicone implant placed in the chin, where bone resorption was intense, increasing the risk of fracture in the region. There was a need to remove the implant, to perform an osteotomy to move the chin forward, and to use an allogeneic graft to fill the spaces and reshape the chin.

\section{Literature Review}

The recommended approach for patients with dentofacial deformities is orthodontic treatment aimed at orthognathic surgery. The indicated surgery of the maxilla and/or mandible varies according to the type and extent of the deformity. This important definition will depend on the soft tissue aesthetic response. In a class III patient, for example, considering the functional aspect, the mandible should be moved backward or the maxilla forward. In both situations, the functional issue would be resolved. What determines whether to move the maxilla or the mandible or perform the combined surgery is the facial aesthetic aspect? Therefore, in many cases, combined surgery is the choice to improve the aesthetic aspect of the face. However, sometimes, to complement facial harmonization, chin surgery is necessary, either for moving the chin forward or backward, reducing or augmenting the chin or correcting asymmetry. The emphasis on the nose/chin relationship is essential for facial harmony and contours. Therefore, 
the excess or deficiency of the chin has a determining influence on this harmony, mainly on the lower third of the face.

To restore a perfect metric balance and general facial harmony, which is determined by the structural balance of several anatomical areas, Ungari, et al., 2015 analyzed 183 male and 338 female patients [1]. All patients underwent orthognathic surgery. The authors sought to determine how this procedure restores maxillofacial deformities and occlusion thanks to the replacement of the mandibular base. The authors concluded that chin surgery is a supportive procedure that helps to maximize the aesthetic results of orthognathic surgery.

Many chin surgery techniques have emerged since its first report, in 1942, by Hofer. In 1948, Rubin introduced the use of alloplastic implants. Since then, several osteotomy designs and implant materials have been developed, increasing the options for changing the dimensions and structures of the lower third of the face. The main indications for chin surgery are: anteroposterior excess, deficiency or asymmetry; vertical excess, deficiency or asymmetry; and transversal excess, deficiency or asymmetry.

Several approaches are described in the literature. Usually, the approach is through osteotomies in the chin and fixation with miniplates and/or screws or steel wires. Other forms of treatment are through biomaterials such as polyethylene or solid silicone, especially in patients with mandibular deficiency treated compensatively, where the profile is impaired. Polyethylene is rigid, comes in various shapes and sizes, and can also be modeled. It needs fixing with screws and has potential for contamination and exposure, but it is a stable material.

Silicone implants have the advantage of less traumatic installation. Also, the procedure can be performed on an outpatient basis, by intra or extra-oral access.

In the treatment of chin deformities, Serna EM, et al., (2008) mention, among other biomaterials, the use of solid silicone (polydimethylsiloxane), a compound derived from silicon available for medical application since 1948 [2]. It is the most widely used alloplastic material worldwide. Unlike the others, silastic does not have pores, thus preventing bacteria from entering its interior, but it also prevents fibrous tissue from growing, decreasing its stability. When the silicone implant is installed, the human body reacts by creating a capsule around it. The fibrous tissue inside the capsule has a reduced blood supply, making it very prone to infection. Silicone implants undergo micro displacement due to the lack of anchoring to the surrounding tissue. This micro displacement explains the chronic periimplantitis that lead to major complications.

Pitanguy, et al., (1986) report a 16-year experience (in a sample of 612 patients) of using silicone implants [3]. The authors point out two main problems: bone absorption by 
compression of the implant, damage to the roots of the teeth and displacement of the implant when not fixed, resulting in loss of result of the projection of the chin and alteration of the mandibular contour. When displaced downward, the implant can change the mentocervical angle, generating a double chin. If displaced upward, the implant changes the labiomental groove, phonation, retention of saliva and the swallowing movement. However, with the author's own technique, the implants showed good results.

Aspects such as low toxicity, low carcinogenic potential, satisfactory bioactivity so that organic rejection does not occur and sufficient stability to support the physiological environment and prevent changes from occurring at the recipient site are crucial for achieving success and longevity in the treatment, according to Ramalho, et al., (2017) [4]. Among the advantages of silicone implants, the authors mention a lower risk of sensory loss and the possibility of removal if the result is unsatisfactory. On the other hand, the disadvantages are: asymmetry, bad positioning, hematoma, seroma, infection, mobility, extrusion and bone resorption. When it is necessary to remove the implant material for any of the reasons mentioned above, lip ptosis can occur due to the loss of the insertion of the mentalis. In these cases, the best option would be to perform the chin surgery through basilar osteotomy. Patients with class II dentofacial deformities, long lower third and labial incompetence seem to be more prone to bone resorption in implants installed in the chin, because this hyper function leads to pressure and the migration of the implant upward over the thinner bone, which predisposes to resorption. In addition, in these patients, implants are more likely to be placed for aesthetic purposes.

Robinson and Shuken, in 1969, reported for the first time the absorption of the mandibular symphysis bone associated with the use of an allogeneic silicone implant, in a sample of 25 patients, of which 11 had resorption [5]. The authors point out that patients undergoing this procedure must be previously informed of the risk of erosion, and must undergo periodic radiological examinations to analyze the region.

Sciaraffia, et al., (2018) analyzed through lateral radiographs of the chin the presence of bone resorption after the insertion of silicone chin implants in 15 patients, all of whom underwent the same surgical technique and were followed up for at least 1 year [6]. Fourteen had bone resorption of a maximum of $2 \mathrm{~mm}$. Despite erosions in most of the sample, all patients remained asymptomatic. The authors propose that each patient reacts differently to the implant and suggest periodic follow-ups to analyze future complications.

In a clinical case, Yamazaki, et al., (2019) reported that placing a silicone implant can cause various complications such as infection, bone resorption and changes in soft tissues [7]. In the case presented by the authors, there was periodontal alteration and dental problem due to infection of the tooth and later of the implant. The patient was treated with an iliac crest graft and a titanium mesh, with a 2-year follow-up. 
Another complication was reported by De Paula DM, et al., (2016), the silicone chin implant had to be removed after exposure to the oral environment. The authors concluded that silicone implants should not be recommended as a long-term aesthetic resolution in young or middleaged patients due to the high rate of bone resorption.

In the study by Isaac J Peled, et al., (1986), 50 children with Down syndrome underwent surgery to install silicone implants in the nasal, cheek, and chin areas [8]. The authors reported that, of these patients, 12 underwent chin implant placement, of which nine had significant bone resorption. Patients were reviewed radiologically 18 to 24 months following the surgical procedures. All of the chin implants were removed, including those from the three patients who had minimal resorption. The implants of the nasal and malar areas did not had changes and this may be explained by the fact that the chin implants lie between bone and active muscles, and great pressure is applied by the muscles to the implants and against the growing bone.

Mario Polo, et al., (2017) discusses the importance of orthodontists regarding the diagnosis and management of bone resorption associated with chin implants [9]. The author reports three patients with bone resorption who were referred for surgical evaluation. He emphasizes that orthodontists often end up seeing bone resorption as an incidental finding on standard radiographs.

\section{Clinical Case Report}

The patient was a 62 year-old woman, without comorbidities, who sought the evaluation of a specialist in oral and maxillofacial surgery after undergoing routine panoramic radiography and reporting discomfort in the anterior mandible to the orthodontist. She reported that 8 years back she underwent surgery to place a silicone chin implant, since she had a class II face with compensated occlusion (Fig. 1). After the clinical evaluation, a computed tomography scan of the implant region was requested. Following the analysis of the images (Fig. 2), removal of the silicone implant was recommended. It was also recommended that the patient undergo an osteotomy for mentoplasty with the need to place an allogeneic graft to fill the defect caused by the implant and to help fix the osteotomized segments. The surgery was performed under general anesthesia in a hospital environment, with an incision in the bottom of the vestibule of the anterior region. The implant was removed (Fig. 3) and significant bone resorption was observed, with an imminent possibility of fracture. Then, vertical markings were performed to assist in repositioning the osteotomized segment to avoid deviation when applying rigid fixation. The allogeneic graft from the PUC-Pr tissue bank was modeled to fill the bone defect and to assist in fixation with a miniplate and screws (Fig. 4). Suture was performed in layers and the wound site was covered with dressing for 5 days to avoid ptosis of the lower lip. Postoperative tomographic follow-up (Fig. 5) and after 4 year (Fig. 6), Twelve-month follow- 
up with return of sensitivity. Frontal view and profile of the patient's face after 4 year of surgery (Fig. 7).

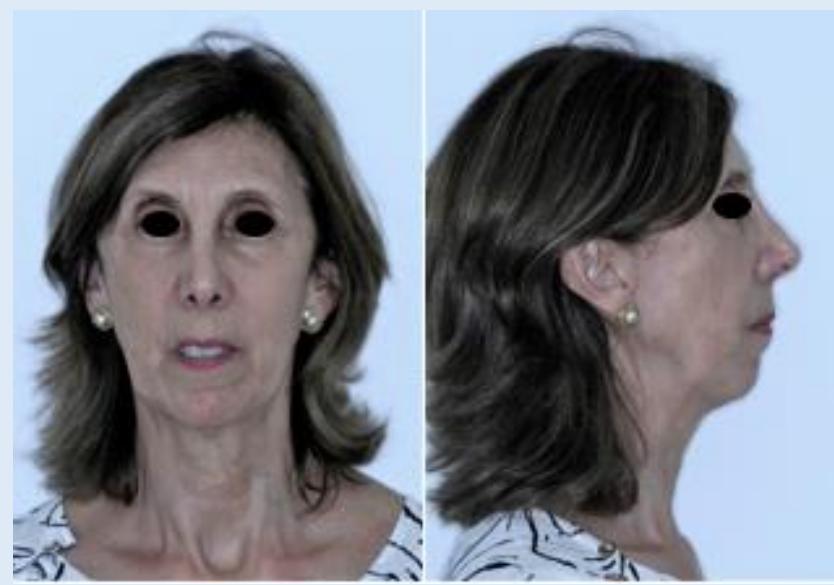

Figure 1: Preoperative front and profile view.

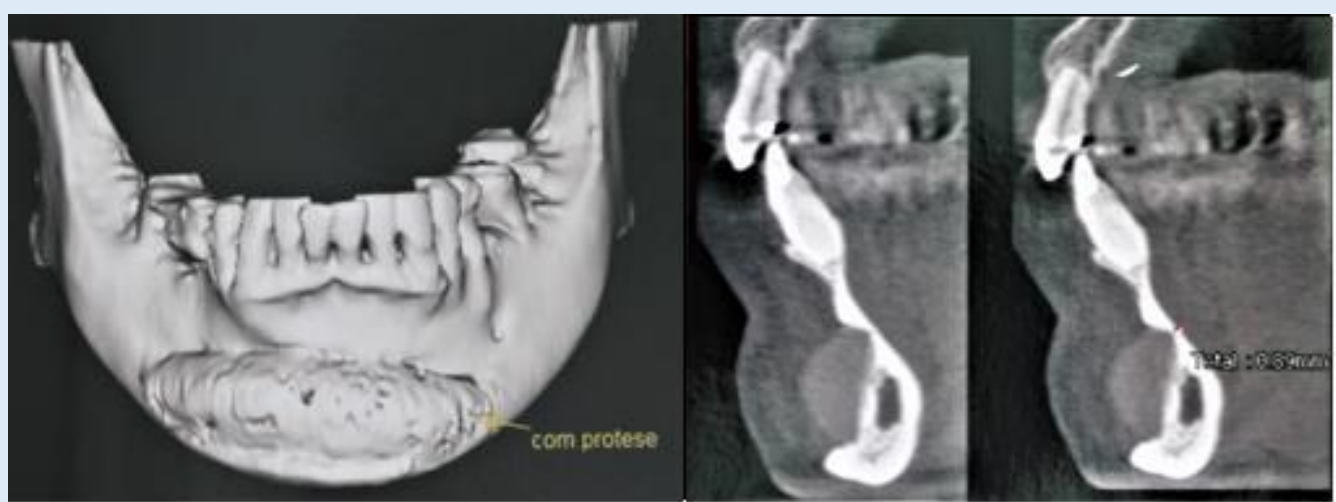

Figure 2: Preoperative CT scan front and profile view.

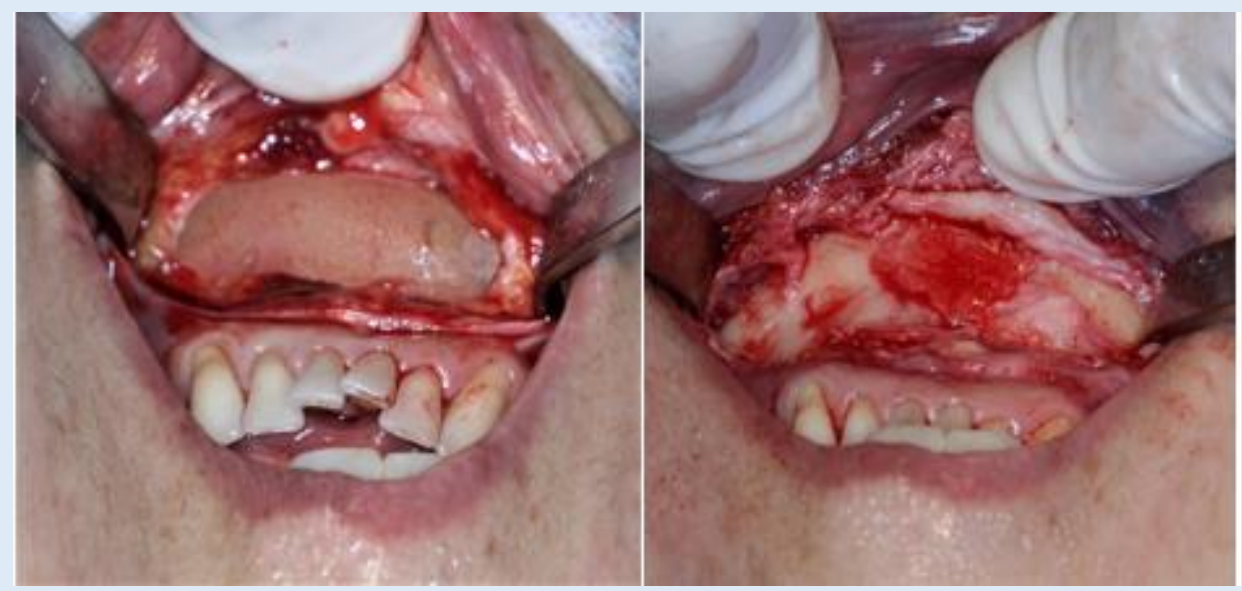

Figure 3: Removal of silicone implant and bone defect.

Carlini JL | Volume 2; Issue 1 (2021) | JDHOR-2(1)-021 | Case Report

Citation: Carlini JL, et al. Surgical Reintervention in the Chin in a Patient with a Silicone Chin Implant

- A Case Report. J Dental Health Oral Res. 2021;2(1):1-8. 


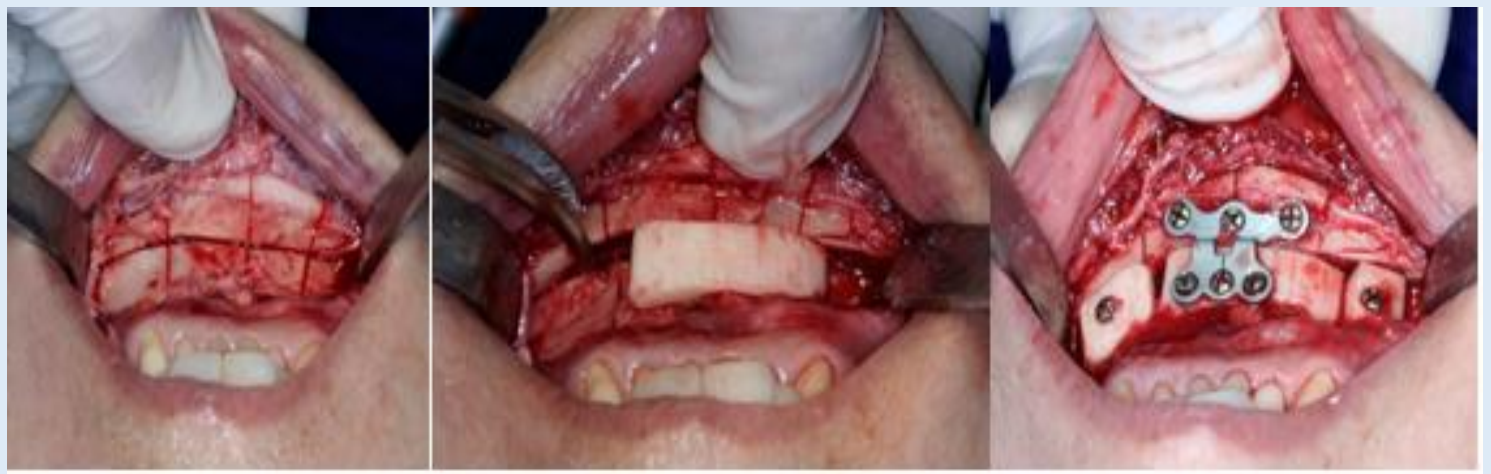

Figure 4: Markings to assist in the repositioning of the chin; adaptation of the allogeneic graft; stabilization of segments and grafts.

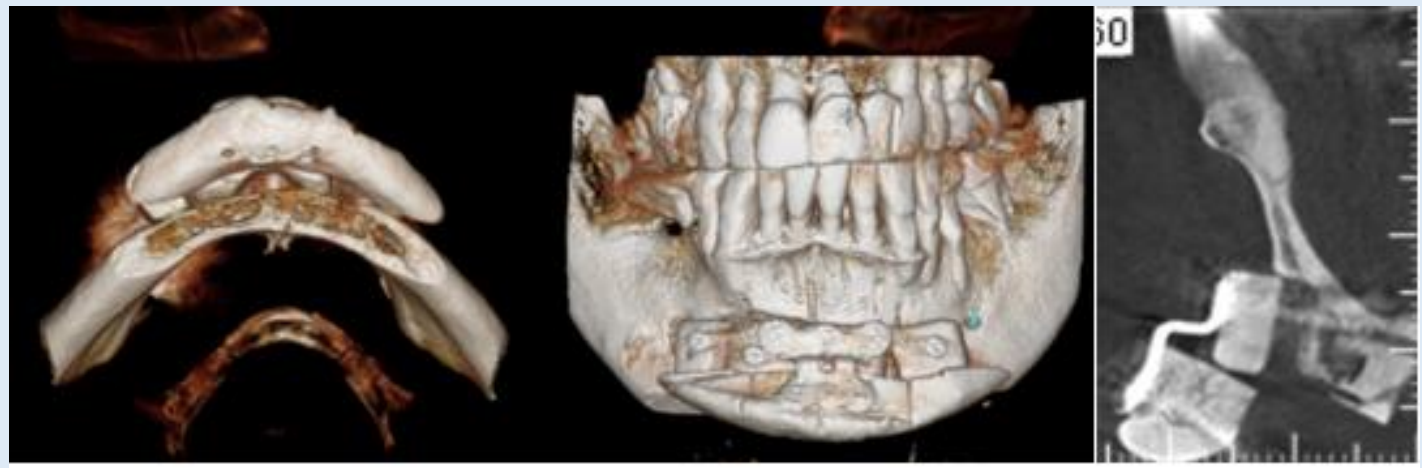

Figure 5: Posperative CT scan axial and frontal and sagittal.
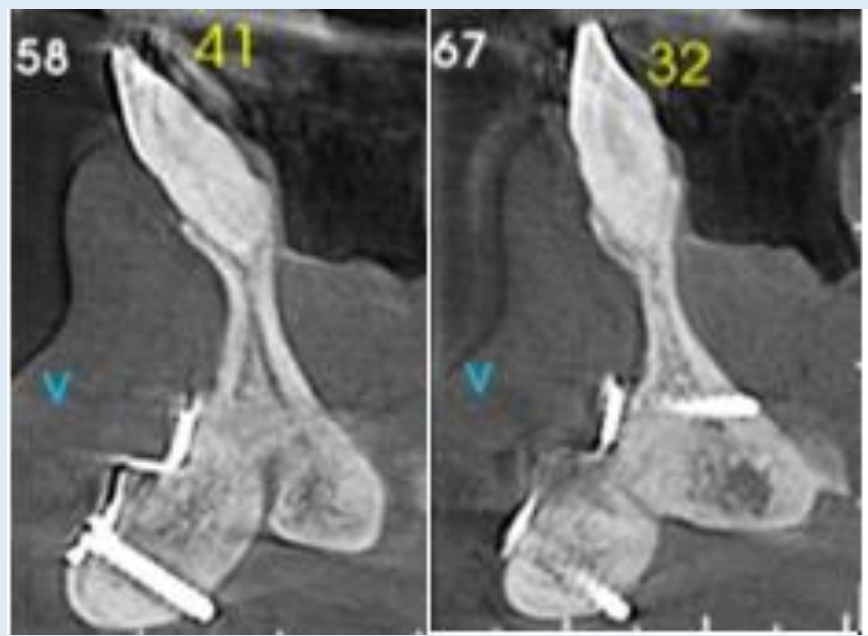

Figure 6: Posperative CT scan after 3 years. 


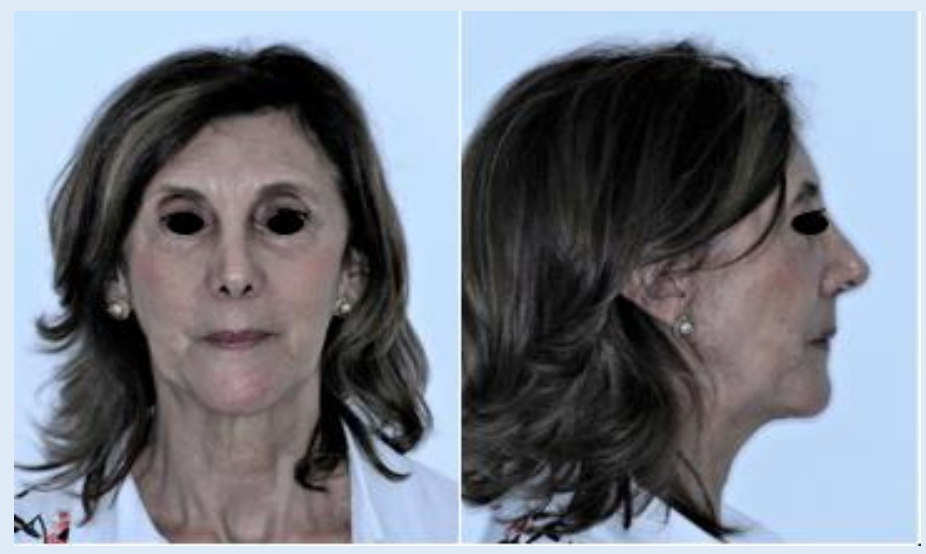

Figure 7: Posperative front and profile view (3years).

\section{Conclusion}

The use of alloplastic materials is an option for the treatment of chin deficiency. However, the soft tissue compression over the years can promote significant resorption of the symphyseal bone. It is important to control installed implants or choose another material or segment osteotomy technique and skeletal fixation. It is observed that the long term use of silicone implants generates bone erosion, causing damage to bone and teeth, in addition to impairing the aesthetic result of the chin.

\section{References}

1. Ungari C, Riccardi E, Reale G, Agrillo A, Rinna C, Mitro V, et al. Aesthetic restoration in maxillo-mandibular malformations: the role of genioplasty. Annali Di Stomatologia. 2015;6(3-4):110.

2. Serna EM, Pliego ES, Ulldemolins NM, Morán AM. Treatment of chin deformities. Acta Otorrinolaringologica. 2008;59(7):349-58.

3. Pitanguy I, Martello L, Caldeira AM, Alexandrino A. Augmentation mentoplasty: a critical analysis. Aesthetic Plastic Surg. 1986;10(1):161-9.

4. Ramalho G, Miranda S, Moreno R, Silva H, Miranda M. Bone reabsorption associated with silicone implants in mentoplasty: a clinical case report. Revista Brasileira de Cirurgia Plástica. 2001;32(2):291-4.

5. Robinson M. Bone resorption under plastic chin implants. J Oral Surg. 1969;27:116-8.

6. Sciaraffia CE, Ahumada MF, Parada FJ, Gonzalez E, Prado A. Bone resorption after use of silicone chin implants, long-term follow-up study with lateral chin radiography. Plastic and Reconstructive Surgery Global Open. 2018;6(7).

7. Yamazaki F, Takahashi K, Aoki A, Yano T, Tajima M, Ito K, et al. Bone resorption after alloplastic chin augmentation found incidentally in a patient with a toothache: Report of a case. J Oral and Maxillofacial Surgery, Medicine and Pathology. 2019;31(4):275-9.

8. Peled IJ, Wexler MR, Ticher S, Lax EE. Mandibular resorption from silicone chin implants in children. J Oral and Maxillofacial Surgeons. 1986;44(5):346-8.

9. Polo M. Bone resorption under chin implants: The orthodontist's role in its diagnosis and management. Am J Orthodontics and Dentofacial Orthopedics. 2017;151(1):201-8. 